\title{
First results from Run-1 of the Extreme Energy Events experiment
}

\author{
M.Abbrescia $^{a b}$, C.Avanzini ${ }^{a c}$, L.Baldini ${ }^{a c}$, R.Baldini Ferroli ${ }^{a d}$, G.Batignani ${ }^{a c}$, \\ G.Bencivenni $^{d}$, E.Bossini $^{a e}$, E.Bressan $^{a f}$, A.Chiavassa $^{g}$, C. Cicalo ${ }^{a h}$, L. Cifarelli ${ }^{a f}$, \\ E.Coccia $^{i}$, A.Corvaglia ${ }^{a l}$, D.De Gruttola ${ }^{a m}$, S. De Pasquale ${ }^{a m}$, A.Di Giovanni ${ }^{n}$, \\ M.D'Incecco $^{n}$, M.Dreucci ${ }^{d}$, F.L.Fabbri $^{d}$, E.Fattibene ${ }^{o}$, A.Ferraro ${ }^{o}$, R.Forster ${ }^{p q}$, \\ V.Frolov $^{r}$, P.Galeotti ${ }^{a g}$, M.Garbini ${ }^{a f}$, G.Gemme $^{r}$, I.Gnesi ${ }^{a g}$, S.Grazzi $^{a s}$, C.Gustavino $^{n}$, \\ D.Hatzifotiadou ${ }^{a f q}$, P.La Rocca ${ }^{a t}$, A.Maggiora ${ }^{g}$, G.Maron $^{o}$, M.N.Mazziotta ${ }^{u}$, \\ S.Miozzi ${ }^{a d i}$, F. Nozzoli ${ }^{w}$, M.Panareo ${ }^{a l}$, M.P.Panetta ${ }^{a l}$, R.Paoletti ${ }^{a e}$, L.Perasso $^{a s}$, F.Pilo $^{a c *}$ \\ , G.Piragino ${ }^{g}$, F.Riggi ${ }^{a t}$, G.C.Righini ${ }^{a}$, A.Rodriguez Rodriguez ${ }^{p q}$, G.Sartorelli ${ }^{a f}$, \\ E.Scapparone $^{f}$, M.Schioppa $^{a z}$, A.Scribano ${ }^{a e}$, M.Selvi $^{f}$, S.Serci $^{g}$, E.Siddi $^{h}$, \\ S.Squarcia ${ }^{s}$, M.Taiuti $^{s}$, G.Terreni $^{c}$, M.C.Vistoli ${ }$ L.Votano $^{n}$, M.C.S.Williams $^{e q}$, S.Zani $^{o}$, \\ A.Zichichi ${ }^{a f q}$ and R.Zuyeuski ${ }^{p q}$ \\ a Museo Storico della Fisica e Centro Studi e Ricerche Enrico Fermi, Roma, Italy \\ b INFN and Dipartimento di Fisica, Universit ̃̃a di Bari, Bari, Italy \\ c INFN and Dipartimento di Fisica, UniversitÃ $\breve{a}$ di Pisa, Pisa, Italy \\ d INFN, Laboratori Nazionali di Frascati, Frascati (RM), Italy \\ e INFN Gruppo Collegato di Siena and Dipartimento di Fisica, UniversitÃ ă di Siena, Siena, Italy \\ f INFN and Dipartimento di Fisica e Astronomia, UniversitÃ $\breve{a ~ d i ~ B o l o g n a, ~ B o l o g n a, ~ I t a l y ~}$ \\ g INFN and Dipartimento di Fisica, UniversitÃă di Torino, Torino, Italy \\ h INFN and Dipartimento di Fisica, UniversitÃă di Cagliari, Cagliari, Italy \\ i INFN and Dipartimento di Fisica, UniversitÃă di Roma Tor Vergata, Roma, Italy \\ l INFN and Dipartimento di Fisica, UniversitÃă del Salento, Lecce, Italy \\ $m$ INFN and Dipartimento di Fisica, UniversitÃ ă di Salerno, Salerno, Italy \\ $n$ INFN, Laboratori Nazionali del Gran Sasso, Assergi (AQ), Italy \\ o INFN CNAF, Bologna, Italy \\ p ICSC World Laboratory, Geneva, Switzerland \\ $q$ CERN, Geneva, Switzerland \\ r JINR Joint Institute for Nuclear Reserch, Dubna, Russia \\ s INFN and Dipartimento di Fisica, UniversitÃă di Genova, Genova, Italy \\ t INFN and Dipartimento di Fisica e Astronomia, UniversitÃ $\breve{a ~ d i ~ C a t a n i a, ~ C a t a n i a, ~ I t a l y ~}$ \\ u INFN, Sezione di Bari, Bari, Italy \\ $\checkmark$ INFN and ASI Science Data Center, Roma \\ z INFN and Dipartimento di Fisica, UniversitÃ $\breve{a}$ della Calabria, Cosenza, Italy \\ E-mail: federico.pilo@pi.infn.it
}


The Extreme Energy Events (EEE) Project is an experiment for the detection of Extensive Air Showers of energy greater than $10^{11} \mathrm{eV}$. It consists of an array of telescopes hosted in High Schools spread on the Italian territory, each made of three Multigap Resistive Plate Chambers very similar to the ones built for the Time Of Flight system of the ALICE experiment at CERN. The telescopes are constructed and managed at CERN by teams of students and teachers: this peculiarity enhances the scientific relevance of its goals with an effective outreach action. The experiment took a first coordinated data taking ("Pilot-Run") in fall 2014 and another ("Run-1") from February to April 2015. About thirty telescopes took data at the same time and more than 5 billions of cosmic ray events have been collected. Data were transmitted to the CNAF, the biggest Italian storage and computing center managed by INFN, to be reconstructed and analyzed. In this presentation an overall description of the experiment will be given and the most recent results will be shown. In particular we have a first set of measurements of the coincidences in pairs of telescopes even more than $1 \mathrm{~km}$ apart, and a study of variations with time of the muon cosmic flux, related on astrophysical phenomena like Forbush decreases.

The 34th International Cosmic Ray Conference,

30 July- 6 August, 2015

The Hague, The Netherlands

${ }^{*}$ Speaker. 


\section{The EEE project}

Having started as a set of seven pilot sites taking data since 2008, the EEE project has evolved into an array of cosmic-ray telescopes distributed in several sites spread out over all the Italian territory, across an area larger than $5 \times 10^{5} \mathrm{~km}^{2}[1,2,3]$.

The main scientific goal of the project is the detection of Extensive Air Showers (EAS) produced by the impact of high-energy primary cosmic rays (of energy greater than $10^{11} \mathrm{eV}$ ) with the atmosphere. EEE is particularly designed for the detection of the muonic component of the EAS, which is a prominent one at ground level, as muons are deeply-penetrating, long-living particles and carry information about energy and nature of the parent (primary) cosmic ray.

Devised in 2004 as a joint scientific and educational initiative by Centro Fermi (Museo Storico della Fisica e Centro Studi e Ricerche "Enrico Fermi"), in collaboration with INFN, CERN and MIUR (the Italian Ministry of Education, University and Research), the EEE project is strongly contributing at introducing a large number of high-school teachers and students to the methods and results of particle and astroparticle physics: the school buildings have been chosen as detector sites, and teams of students are directly involved in the construction, installation, operation and data analysis of the experiment, under the supervision of researchers from scientific Institutions.

The EEE net has been constantly extended during the past years, reaching the present configuration with more than 50 operative sites (47 in high schools +5 in Labs), organized as clusters or single stations.

\section{The EEE detector}

The design of the EEE telescope allows muons from EAS to be detected with high efficiency and back-tracked with good angular resolution. Each telescope is composed by three MRPC chambers of $160 \times 80 \mathrm{~cm}^{2}$ size, a simplified and larger version of the detectors developed for the time-offlight system of the Alice experiment. Each MRPC consists of six gas gaps obtained interleaving two glasses, coated with resistive paint and acting as electrodes, with a stack of five floating glasses. Commercial nylon fishing line ( $300 \mu \mathrm{m}$ thick) is used as spacer between glasses. The chambers are filled with a mixture of $98 \%$ of $\mathrm{C} 2 \mathrm{H} 2 \mathrm{~F} 4$ and $2 \%$ of SF6 and are operated in avalanche mode.

The MRPC pick-up electrodes are segmented into 24 strips read out at the two ends. This strip configuration is used to provide two-dimensional information when a particle crosses the chamber: one coordinate is given by the fired strip, while the second is obtained by the time difference of the arrival signals at the opposite ends in each strip. The strips are read out by front-end (FEA) cards, which incorporate an ultra-fast and low-power amplifier/discriminator ASIC specifically designed for MRPC operation [4]. From the front-end cards a total of 144 LVD digital signals are fed into two commercial multi-hit TDCs which provide time measurements with a 100 ps precision in a time window of $500 \mathrm{~ns}$; this solution provides a value of about $70 \mathrm{ps}$ for the time resolution and $0.7 \mathrm{~cm}$ for the spatial resolution along the readout strips direction, while in the transverse direction the measured spatial resolution is about 0.25 in units of strips, corresponding to $0.63 \mathrm{~cm}$ [5]. Uncertainties in the position of the hits along the muon track directly affect the reconstruction of the particle direction. The telescope angular resolution can be estimated from two-tracks events, in which two muons belonging to the same shower front pass through the telescope; fig. 1 shows 
the 3D angular distance of two-track events: the green area, typically contained in a 6-7 degree interval, represents the $68 \%$ of the events.

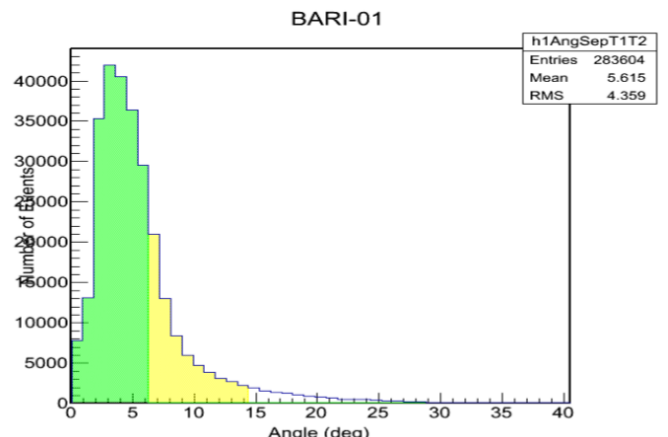

Figure 1: Angular resolution of a telescope estimated from two track events; the green area contains $68 \%$ of the events.

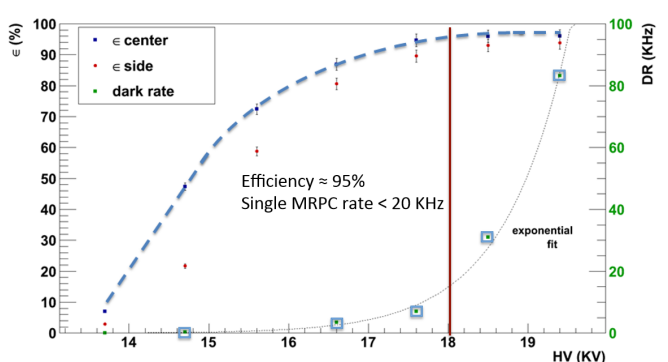

Figure 2: Efficiency and counting rate of a typical MPRC detector developed for the EEE telescope.

The telescope data acquisition system is VME based, controlled by a LabView interface running on a PC connected to the VME crate via a USB-VME bridge. Along with the TDC's and the bridge, two more boards realizing trigger logic and GPS interfacing complete the set of board hosted in the VME crate. The trigger is generated starting from the signals produced in the frontend for each side of the MRPC's as OR of all the 24 input channels; these 6 signals are combined according to AND logic, thus requiring a triple coincidence of signals from the three MRPC's: this assures a negligible level of spurious events even if the dark current rate of each chamber is generally well above $10 \mathrm{kHz}$ (see fig. 2). The output signal from the trigger board is sent to the "GPS-interface" board where it is combined with the PPS signal produced by the GPS receiver and then distributed to the TDC board; on PPS arrival the GPS-interface also produce a reset pulse for the counters used by TDC's for time measurements and this allow a synchronization of distant EEE stations with a precision lower than $50 \mathrm{~ns}$.

\section{Centralized runs}

In 2014 the EEE project received a new boost with the organization of simultaneous and centralized data taking with the whole telescope array. Data coming from all the sites were stored in a unique storage center: this unified operation mode made comparison studies between different sites much easier, resulting in higher and more equalized performances of all the detectors network. Morevoer, a common set of hardware and software requirements that the telescopes have to fulfill to be included in the data taking, has been identified: this ensures that all the detectors, assembled and commissioned over a period of several years, are constantly mainteined and upgraded.

Data acquisition has been arranged in "runs" as in typical high energy accelerator experiments: after a week long period of setting up and commissioning, all the telescopes were set in continuous data taking mode (24 hours/day). Daily-based shifts have been organized to constantly monitor detector performances, both locally by group of students specifically instructed, and remotely by EEE researchers using on-line software tools; in case of problems, remote shifters get in contact 
with a group of referent researchers that act as a liaison with each school and supervised telescope control operations. During data acquisition raw files containing 50,000 events are continuosly recorded on the local computer disks. The peculiarity of the location of the detectors, as well as the considerable inter-distances among different sites, required non-standard innovative solutions in terms of data management and quality monitoring to make centralized runs possible.

\subsection{Data management}

The EEE project joined the CNAF cloud facility to create its own data collection center. The CNAF cloud provides a flexible environment based on OpenStack and virtualization which allows to allocate on demand resources adapted to the need of the experiment and to collect data from the telescopes which are distributed in a wide territory.

During coordinated runs all the schools have been connected/authenticated at CNAF in order to transfer data using a BitTorent technology. To realize this goal a BitTorrent Sync client (Win OS) was installed in each school, and a dedicated front-end at CNAF received all the data, with a total bandwidth of $300 \mathrm{kB} / \mathrm{s}$ that is required to collect $5 \div 10 \mathrm{~TB}$ expected per year. All the data collected are considered as custodial and for this reason they are stored also on tape. In fig. 3 the general architecture for the EEE data flow is shown.

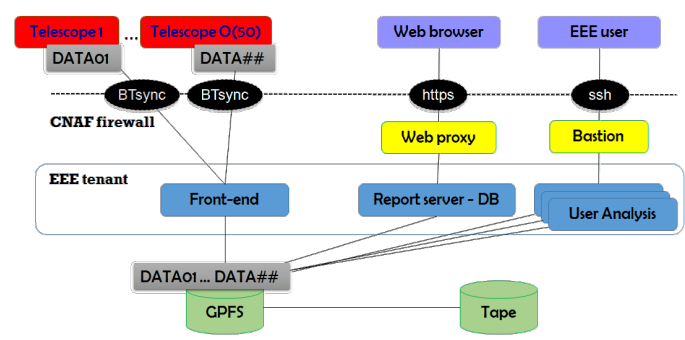

Figure 3: Architecture of the EEE tenant at CNAF.

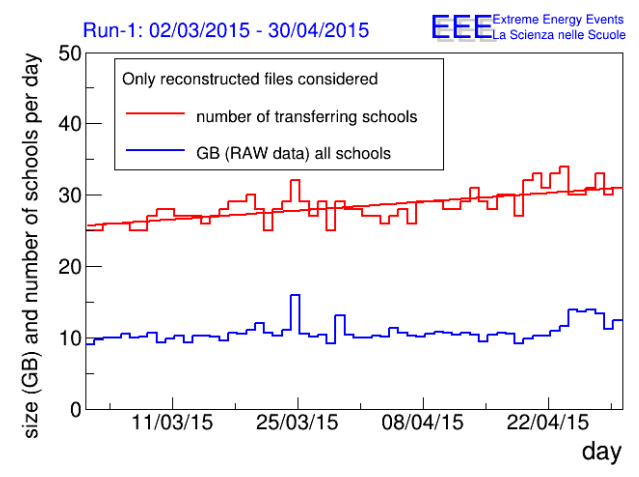

Figure 4: Statistics for the EEE Run-1 in 2015.

\subsection{Reconstruction and quality monitoring}

Data transferred and stored at CNAF are analyzed and reconstructed by a fully automated procedure. This point is crucial because all the schools have to be monitored also remotely to act promptly in case of problems. This is addressed by automatic agents, running in a CNAF node dedicated to this issue, which are able to identify the arrival of a new file and then to trigger the reconstruction. A MySql database is deployed to trace all the actions performed on each single file and the main parameters resulting from the reconstruction.

A unique reconstruction algorithm is applied to all raw data. It starts with a preliminary evaluation of the hit multiplicity, selecting only hits whose coordinates are comprised in the strip geometry; good hits are then associated in clusters centered in the average position of the fired strips. A simple tracking procedure is then applied to the set of reconstructed clusters, using a threedimensional fit, which provides the incoming direction of each muon. Additional track quality 
checks are also applied, to consider only tracks which have a correct time of flight between the top and the bottom chambers, a correspondingly correct track length and the lowest $\chi^{2}$ among all the possible combinations. These cuts help to keep the amount of spurious coincidences below $0.1 \%$. Both single-track events and two-track events (which represent a few percent of the total) are reconstructed whenever possible. Good performing stations usually show a fraction of tracks with $\chi^{2}<10$ above $90 \%$. Once the run is processed, a DST (Data Summary Tape) output is created and many quality plots are made available and published in the web page devoted to the monitor [6]. This "quasi-online" monitor page displays the global status of each telescope and if necessary produces alerts in case of faults; a set of links gives the researchers access to a complete set of run-by-run plots, as well as to daily summary reports (fig. 5), thus allowing a fast diagnosis of issues that could arise.

EEE DQM summary report
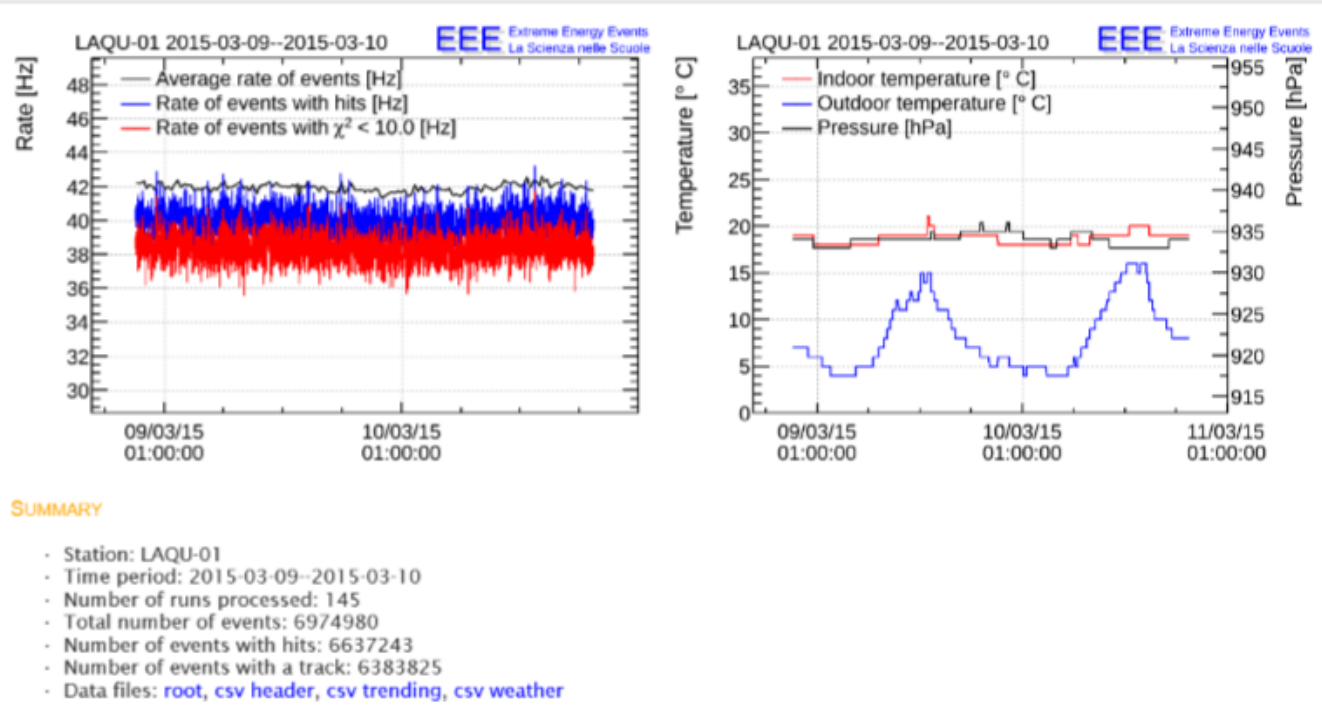

Figure 5: Typical daily reports of an EEE telescope data quality.

In parallel with the reconstruction, a cluster of CNAF analysis nodes is reserved to EEE users via virtual node, constructed on a dedicated immage of the Operating System selected for the experiment (SL6). The EEE users authenticated at CNAF can access data (both RAW and DST files), as well the software of the experiment, via a gpfs filesystem.

\section{First results from coordinated runs}

After a pilot Run in November 2014, from February 23 to April 30, 2015, for the first time 35 of the EEE telescopes have been acquiring data at the same time. Throughout these 45 days, identified as Run-1, students and teachers at each station, as well as researchers of the EEE Project have been constantly monitoring and controlling the telescopes status. During the pilot run and Run-1, about 7 billion cosmic rays were collected, corresponding to 2 TB data transferred at CNAF. In fig. 4 a summary of the data flow performances during Run-1 is reported. The analysis activity is 
currently in progress and focused on several items like coincidence searches (between two and three stations), time variations of the muon flux, East-West asymmetry, cosmic ray anisotropy, upwardgoing particles, observation of the moon shadow. Here we will briefly show some preliminary results from the first two topics.

\subsection{Extensive atmospheric showers detection}

The relative distances of the EEE network telescopes range from a few hundred meters for clusters of 2, 3 and 4 telescopes in the same city, to more than $1000 \mathrm{~km}$ for the farthest stations. Being EAS detection the main goal of the EEE scientific program, a systematic study on the number of coincidences detected by pairs of EEE telescopes is extensively investigated, making use of sophisticated Monte Carlo simulation programs (like CORSIKA or COSMOS), e.g. to compute the expected number of coincidences as a function of the distance between the two sites [7].

The first detection of EAS performed at L'Aquila, as well as the results from two $0.5 \mathrm{~km}$ far away telescopes in the Cagliari site, have been already reported elsewhere [8] [9]. From all measurements it is evident that the coincidence rate diminishes as the distance between the telescopes increases, as expected: from a certain distance on, to distinguish the signal among the background level is mandatory to operate the telescopes with the maximum duty cycle possible. Analysing jointly pilot-RUN and RUN-1 with their huge ammount of high-quality data, resulted in the possibility to move the limit of detectable coincidences beyond the $1 \mathrm{~km}$ limit. Fig. 7 shows the first detection of coincident events in the time-difference spectrum obtained between two telescopes in Savona, where the distance of is about $1.2 \mathrm{~km}$; the significance is $S / \sqrt{(} S+B)=5.1$.

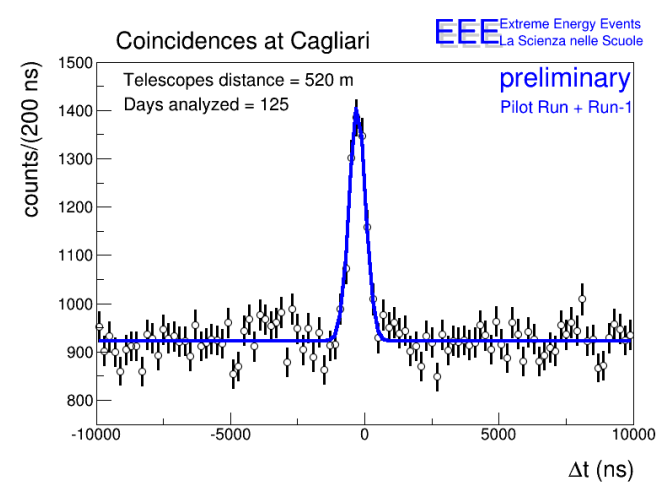

Figure 6: Observations of coincidences between stations in Cagliari.

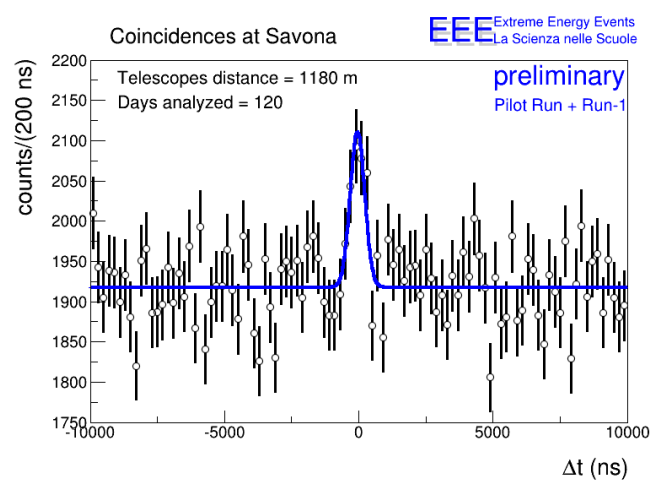

Figure 7: Observations of coincidences between stations in Savona.

To achieve the result shown, a careful investigation of techniques for effectively rejecting the background of accidentals has been exploited. EEE telescopes can detect the direction of the shower secondaries. Such a feature allows to correct, event by event, the time delay between two telescopes because of the propagation of the wave front of the shower. Under the approximation of flat shower front, the time difference depends on the distance $\Delta \mathrm{L}_{\text {school }}$ between the stations and on the angle between the EAS axis and the imaginary line joining the two stations $\left(\Delta \phi_{\text {school }}-\phi\right)$ as reported in fig. 8 . This correction is very important when looking at coincidences at very large distances, since above $1 \mathrm{~km}$ the time delay may be of the order of few microseconds. 


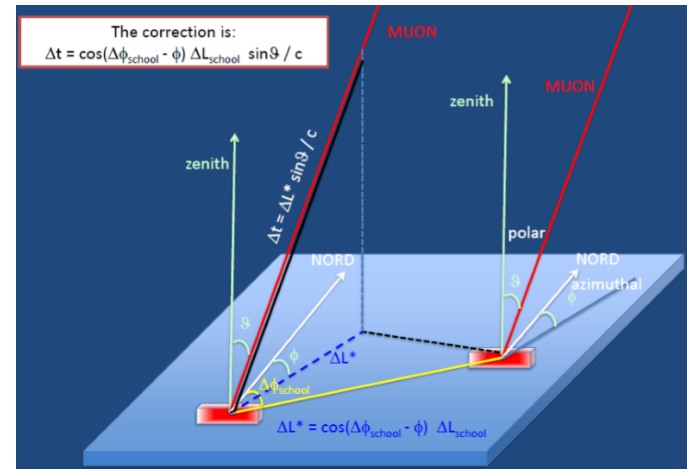

Figure 8: Time delay between two telescopes because of the propagation of the EAS front.

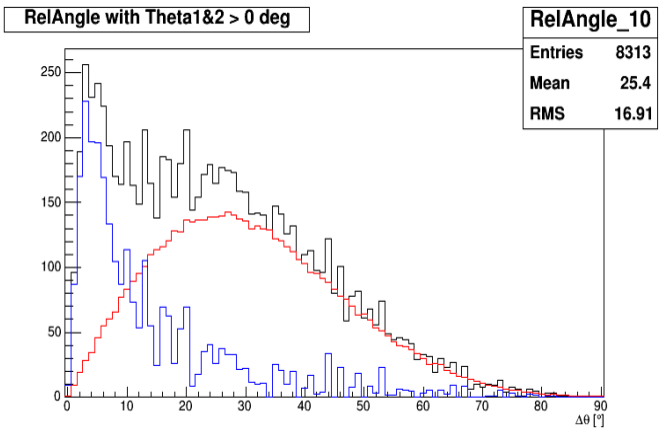

Figure 9: Relative angle distribution between two coincident events.

The signal-to-noise ratio can be further improved by selecting events where tracks have a small relative angle, because muons belonging to the same EAS are nearly parallel. Fig. 9 shows, for the L'Aquila site, the distribution of the relative angle between two tracks collected in different telescopes: both the shape of the background, computed for events with large time difference, as well as that of the signal are shown. As expected, the angles between coincident events are small even if the distribution is folded with the angular resolution of the instrument; putting a limit on the maximum angle between two tracks can cleary enhance the signal evidence.

\subsection{Study of cosmic rays flux}

Designed for a 24/7 operation, the EEE array is a powerful tool for monitoring the cosmic-ray flux, usually affected by periodic and non-periodic variations. The high sensitivity of the telescopes allows to detect small fluctuations of the local muon flux, e.g. due to meteorological effects or to non-periodic solar phenomena. Very promising seems to be the possibility of studying these variations as a function of the arrival direction at different coordinates and with precise timing.

Details of flux variation analysis of the data collected during the coordinated runs are described in detail in [10]. In summary, using the local pressure measurement, acquired together with the data and trasferred to CNAF, the rate of each telescope has been corrected for variations induced by atmospheric pressure changes. Then, using a semi-automatic analysis chain and choosing only telescopes with stable acquisition, it was possible to detect a variation of the order of $5 \%$ on November 10, 2015 10. This variation, known as Forbush decrease, observed at the same time by six EEE stations covering almost the whole latitude and longitude range, is in good agreement with the OULU University neutron monitor observation.

\section{Conclusion}

The EEE Project, being built and operated with a coordinate effort of researchers from scientific institutions and high-school students and teachers, has proven to combine an effective outreach action with significant scientific outcomes.

The coordinated operation of telescopes at sites spread over all the italian territory required non-standard innovative solutions for data management and quality monitoring. During the cen- 


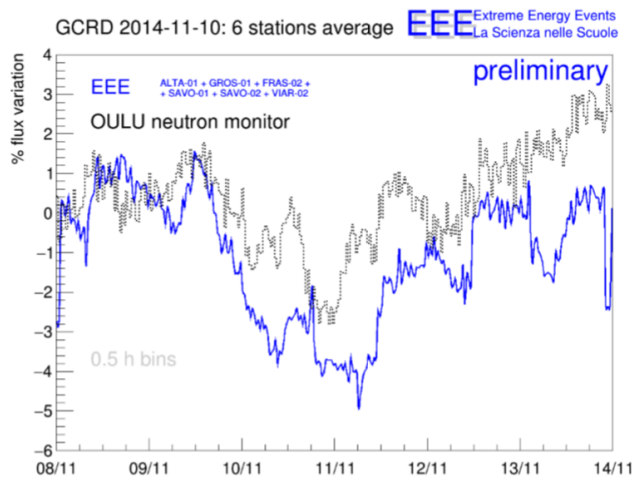

Figure 10: Observation of a Forbush decreases during the EEE pilot run.

tralized runs more than 7 billions of cosmic ray events have been collected, then transferred to a central facility to be reconstructed and analyzed with a unified approach. The analysis is in progress and has given by this time significant results on the performance of the detector array, confirming its capability of studying the main characteristics of the EAS. This already allowed the observation of significant decreases of the cosmic-ray flux in corralation with solar phenomena, and as the statistics increases new perspectives open up for more detailed results on cosmic-ray related topics, such as the observation of upward-going particles, of the moon shadow, of the expected East-West asymmetry and the existence of possible anisotropies in the cosmic ray flux.

\section{References}

[1] Centro Fermi web site: http://www.centrofermi.it/eee.

[2] A. Zichichi, Progetto "La Scienza nelle Scuole" - EEE: Extreme Energy Events (Società Italiana di Fisica, Bologna, 2004) 2nd edition (2005), 3rd edition (2012).

[3] M. Abbrescia et al., Extreme Energy Events Project: construction of the detectors and installation in Italian High Schools, Nucl. Instrum. Meth. A 588 (2008) 211.

[4] F. Anghinolfi, P. Jarron, A. N. Martemyanov, E. Usenko, H. Wenninger, M. C. S. Williams and A. Zichichi, NINO: An ultra-fast and low-power front-end amplifier/discriminator ASIC designed for the multigap resistive plate chamber, Nucl. Instrum. Meth. A 533 (2004) 183.

[5] M. Abbrescia et al., The EEE Project: cosmic rays, multigap resistive plate chambers and high school students, JINST 7 P11011

[6] EEE Monitor web site: http://eee.centrofermi.it/monitor.

[7] M. Abbrescia et al., Cosmic rays Monte Carlo simulations for the Extreme Energy Events Project, Eur. Phys. J. Plus 129 (2014) 166.

[8] M. Abbrescia et al., First detection of extensive air showers with the EEE experiment, Nuovo Cim. B125(2010) 243 .

[9] M. Abbrescia et al., D. D. Gruttola et al., A multigap resistive plate chamber array for the Extreme Energy Events project, JINST 9 (2014) 10, C10024.

[10] M. Abbrescia et al., Results from the observations of Forbush decreases by the Extreme Energy Events experiment, this conference proceedings. 